\title{
CP violating asymmetries in the flavour changing single top quark production
}

\author{
A T Alan, A Senol and A T Tasci \\ Department of Physics, Abant Izzet Baysal University, 14280 Gölköy, Bolu, Turkey \\ E-mail: alan@ibu.edu.tr
}

\begin{abstract}
.
$\mathrm{CP}$ violating effects in the single top quark production via flavour changing neutral current (FCNC) reactions $e^{+} e^{-} \rightarrow t \bar{q}$ and $e^{+} e^{-} \rightarrow \bar{t} q$ (here $q$ refers to charm and up quarks) are studied. The effective Lagrangian description of the FCNC interactions is used. A numerical analysis is performed for some next linear colliders. CP violating asymmetries for the number of $q$ and $\bar{q}$ quarks are obtained to be of order $10^{-2}-10^{-3}$ depending on the CM energy.
\end{abstract}

\section{Introduction}

One of the major goals of the next linear colliders (NLC) will be searching for flavour changing processes. Within the standard model (SM) these processes occur at the oneloop level and are unobservably small [1]. Thus any signal of such reactions will be a clear evidence of new physics beyond the SM. The top quark with its large mass seems to play the leading role to provide useful information on new physics. Searching for CP violation in top sector seems especially promising [2]. For detailed analyzes of CP asymmetries in single top production within the minimal supersymmetric model (MSSM) see 3 . On the flavour changing top-charm (or top-up) transitions, there are lots of theoretical studies in the literature. Two popular models allowing such transitions are multi-Higgs-Douplet models [4] and Supersymmetry [5]. Another possible manifestation of these kind of new interactions in the top sector is to alter its couplings to the other known particles, which is called as effective Lagrangian description [6, 7]. In this approach, deviations from the SM for the flavour changing vertices are described by a linear effective Lagrangian containing a series of effective operators whose coefficients are suppressed by power of $1 / \Lambda$, where $\Lambda$ is a high mass scale up to which the effective theory is assumed to hold. For the flavour changing processes, the lowest dimension gauge invariant operators built from SM fields are dimension six, but after spontaneous symmetry breaking the effective Lagrangian induces the dimension five operators [6],

$$
\begin{aligned}
\mathcal{L}= & e e_{q} \bar{t} \frac{i \sigma_{\mu \nu} q^{\nu}}{\Lambda}\left(\kappa_{\gamma}-i \widetilde{\kappa}_{\gamma} \gamma_{5}\right) c A^{\mu} \\
& +\frac{g}{2 \cos \theta_{W}} \bar{t}\left[\gamma_{\mu}\left(v_{Z}-a_{Z} \gamma_{5}\right)+\frac{i \sigma_{\mu \nu} q^{\nu}}{\Lambda}\left(\kappa_{Z}-i \tilde{\kappa}_{Z} \gamma_{5}\right)\right] c Z^{\mu}+\text { h.c. }
\end{aligned}
$$


where $q$ is the momentum of the exchanged gauge boson, $\theta_{W}$ is the Weinberg angle, $e$ and $g$ denote the gauge couplings relative to $U(1)$ and $S U(2)$ symmetries respectively,

$e_{q}$ denotes the electric charge of up-type quarks, $A^{\mu}$ and $Z^{\mu}$ the fields of the photon and $Z$ boson.

In this paper, we investigate the $\mathrm{CP}$ asymmetries in the FCNC single top quark production. More precisely, we consider the two CP conjugate flavour changing processes $e^{+} e^{-} \rightarrow t \bar{q}$ and $e^{+} e^{-} \rightarrow \bar{t} q$ (here q refers to charm or up quarks). For numerical evaluations, the parameters of the three high energy linear colliders (LC) LEP II, TESLA and CLIC are used [8, 9].

\section{Derivation of the $\mathrm{CP}$ violating asymmetry}

Considering only the CP violating $\tilde{\kappa}_{\gamma}$ and $\tilde{\kappa}_{Z}$ terms in the effective Lagrangian, contribution of the CP conserving couplings to the asymmetry are less than $0.3 \%$, the top decays $t \rightarrow q \gamma$ and $t \rightarrow q Z$ are written as

$$
\begin{aligned}
\Gamma(t \rightarrow q \gamma) & =\frac{\alpha e_{q}^{2} \tilde{\kappa}_{\gamma}^{2}}{\Lambda^{2} m_{t}^{3}}\left(m_{t}^{2}-m_{q}^{2}\right)^{3}, \\
\Gamma(t \rightarrow q Z) & =\frac{g_{z}^{2}\left(m_{t}^{2}-M_{Z}^{2}\right)^{2}}{32 \pi \Lambda^{2} m_{t}^{3} M_{Z}^{2}} \\
& \times\left[\left(m_{t}^{2}+2 M_{Z}^{2}\right)\left[\left(a_{Z}^{q}\right)^{2}+\left(v_{Z}^{q}\right)^{2}\right] \Lambda^{2}+\tilde{\kappa}_{Z}^{2} M_{Z}^{2}\left(2 m_{t}^{2}+M_{Z}^{2}\right)\right]
\end{aligned}
$$

where $a_{Z}^{q}=\frac{1}{2}$ and $v_{Z}^{q}=\frac{1}{2}-\frac{4}{3} \sin ^{2} \theta_{W}$.

By using the $95 \%$ C.L. limits of $B R(t \rightarrow q \gamma)<0.032$ and $B R(t \rightarrow q Z)<0.33$ [10], we obtain that while $\tilde{\kappa}_{\gamma}$ is real, $\tilde{\kappa}_{Z}$ is pure imaginary and the restrictions on the couplings are as follows:

$$
\left|\tilde{\kappa}_{\gamma}\right|<0.28 \text { and }\left|\operatorname{Im} \tilde{\kappa}_{Z}\right|<0.58 \text {. }
$$

In obtaining these restrictions we have taken $\Lambda=m_{t}$.

To estimate the possible size of the effect, we consider the following CP-violating quantity :

$$
A_{C P}=\frac{\sigma(\bar{t} q)-\bar{\sigma}(t \bar{q})}{\sigma(\bar{t} q)+\bar{\sigma}(t \bar{q})}
$$

where $\sigma(\bar{t} q)$ and $\bar{\sigma}(t \bar{q})$ are the total cross sections for the processes $e^{+} e^{-} \rightarrow \bar{t} q$ and $e^{+} e^{-} \rightarrow t \bar{q}$, respectively. The total cross section for the process $e^{+} e^{-} \rightarrow \bar{t} q$ is obtained as

$$
\sigma=\sigma_{\gamma}+\sigma_{Z}+\sigma_{i n t}
$$

where $\sigma_{\gamma}, \sigma_{Z}$ are the cross sections for $s$ channel $\gamma$ and $Z$ exchange processes, respectively. $\sigma_{\text {int }}$ corresponds to the interference term of the amplitude. These are given by 


$$
\begin{aligned}
\sigma_{\gamma}= & \frac{2 \pi \alpha^{2} e_{q}^{2} \tilde{\kappa}_{\gamma}^{2}}{3 \Lambda^{2} s^{3}}\left[s^{3}-3 s m_{t}^{4}+2 m_{t}^{6}\right], \\
\sigma_{Z} & =\frac{1}{16 \pi s^{2}} \frac{g_{z}^{4}\left[\left(a_{Z}^{e}\right)^{2}+\left(v_{Z}^{e}\right)^{2}\right]\left(m_{t}^{2}-s\right)^{2}}{24\left[\left(s-M_{Z}^{2}\right)^{2}+\Gamma_{Z}^{2} M_{Z}^{2}\right]} \\
& \times\left[\left(m_{t}^{2}+2 s\right)\left[\left(a_{Z}^{q}\right)^{2}+\left(v_{Z}^{q}\right)^{2}\right]+\frac{\left(\operatorname{Im} \tilde{\kappa}_{Z}\right)^{2} s}{\Lambda^{2}}\left(2 m_{t}^{2}+s\right)\right], \\
\sigma_{i n t} & =\frac{\alpha e_{q} \tilde{\kappa}_{\gamma} g_{z}^{2} v_{Z}^{e}\left(m_{t}^{2}-s\right)^{2}}{12 \Lambda^{2} s^{2}\left[\left(s-M_{Z}^{2}\right)^{2}+\Gamma_{Z}^{2} M_{Z}^{2}\right]} \\
& \times\left[3 a_{Z}^{q} m_{t} \Lambda\left(M_{Z}^{2}-s\right)+\operatorname{Im} \tilde{\kappa}_{Z}\left(2 m_{t}^{2}+s\right) \Gamma_{Z} M_{Z}\right] .
\end{aligned}
$$

where $a_{Z}^{e}=-\frac{1}{2}$ and $v_{Z}^{e}=-\frac{1}{2}+2 \sin ^{2} \theta_{W}$. The total cross section for the CP conjugate process $e^{+} e^{-} \rightarrow t \bar{q}$ differs only in the interference term, that is,

$$
\bar{\sigma}_{\gamma}=\sigma_{\gamma} \quad \bar{\sigma}_{Z}=\sigma_{Z}
$$

and

$$
\begin{aligned}
\bar{\sigma}_{i n t} & =\frac{\alpha e_{q} \tilde{\kappa}_{\gamma} g_{z}^{2} v_{Z}^{e}\left(m_{t}^{2}-s\right)^{2}}{12 \Lambda^{2} s^{2}\left[\left(s-M_{Z}^{2}\right)^{2}+\Gamma_{Z}^{2} M_{Z}^{2}\right]} \\
& \times\left[3 a_{Z}^{q} m_{t} \Lambda\left(s-M_{Z}^{2}\right)+\operatorname{Im} \tilde{\kappa}_{Z}\left(2 m_{t}^{2}+s\right) \Gamma_{Z} M_{Z}\right] .
\end{aligned}
$$

\section{Numerical results}

The CP asymmetries are evaluated for LEP II with $\sqrt{s}=200 \mathrm{GeV}$, TESLA with $\sqrt{s}=500 \mathrm{GeV}$ and CLIC with $\sqrt{s}=1 \mathrm{TeV}$. With the increasing CM energy, the ability of a collider to probe the existence of FCNC couplings is improved. But the sensitivity to these couplings scales with the integrated luminosity $L$ approximately as $1 / \sqrt{L}$. As an example in figures 1(a) and 1(b) we display the sensitivity to the couplings as a function of integrated luminosity at $\sqrt{s}=500 \mathrm{GeV}$. In figure 2 the asymmetry is displayed as a function of the CM energy in the range of 200 - $1000 \mathrm{GeV}$. In figures [3] 4] and [5] the CP asymmetries are shown also as a function of couplings $\tilde{\kappa}_{\gamma}$ and $\operatorname{Im} \tilde{\kappa}_{Z}$ for the CM energies $200 \mathrm{GeV}, 500 \mathrm{GeV}$ and $1000 \mathrm{GeV}$, respectively. If these couplings are saturated to their upper bounds given in equation (4) the following values are obtained for three different $\Lambda$ values:

\begin{tabular}{l|lll} 
& $\Lambda=175 \mathrm{GeV}$ & $\Lambda=500 \mathrm{GeV}$ & $\Lambda=1000 \mathrm{GeV}$ \\
\hline$\sqrt{s}=200 \mathrm{GeV}$ & $\mathrm{A}=1.84 \times 10^{-2}$ & $\mathrm{~A}=1.39 \times 10^{-2}$ & $\mathrm{~A}=7.95 \times 10^{-3}$ \\
$\sqrt{s}=500 \mathrm{GeV}$ & $\mathrm{A}=9.79 \times 10^{-3}$ & $\mathrm{~A}=1.45 \times 10^{-2}$ & $\mathrm{~A}=1.09 \times 10^{-2}$ \\
$\sqrt{s}=1000 \mathrm{GeV}$ & $\mathrm{A}=3.22 \times 10^{-3}$ & $\mathrm{~A}=7.17 \times 10^{-3}$ & $\mathrm{~A}=8.16 \times 10^{-3}$
\end{tabular}

The total cross section for the process $e^{+} e^{-} \rightarrow t \bar{q}$, with $\sqrt{s}=500 \mathrm{GeV}$, as a function of the couplings is plotted in figure 6. In all figures we have taken $\Lambda=m_{t}$. 


\section{Conclusions}

We have studied the CP asymmetries in single top production in $e^{+} e^{-}$collisions via FCNC couplings. We used a model independent effective Lagrangian approach to describe these couplings. In this description, the effects turn out to be of the order of $10^{-2}-10^{-3}$. The total cross section for $e^{+} e^{-} \rightarrow t \bar{q}$ is $\sim 330 \mathrm{fb}$ which means $\sim 10^{5}$ events for TESLA with an integrated luminosity of $300 \mathrm{fb}^{-1}$. Therefore, an asymmetry of the order of $10^{-2}$ is to be detectable at TESLA. Finally, similar discussions on the observability of the asymmetries can be done for other future linear colliders, e.g. for CLIC.

\section{Acknowledgments}

This work was supported in part by Abant Izzet Baysal University Research Fund.

\section{References}

[1] Ganapathi V et al 1983 Phys. Rev. D27 579 Buchmüller W and Gronau M 1989 Phys. Lett. B220 641 Fritzsch H 1989 Phys. Lett. B224 423 Huang C S, Wu X H and Zhu S H 1999 Phys. Lett. B452 143

[2] Atwood D, Bar-Shalom S, Eilam G and Soni A 2001 Phys. Rept. 3471

[3] Bar-Shalom S, Atwood D and Soni A 1998 Phys. Rev. D57 1495 Christova E 1999 Int. J. Mod. Phys. A14 1

[4] Atwood D, Reina L and Soni A 1997 Phys. Rev. D55 3156 Bar-Shalom S, Eilam G, Soni A and Wudka J 1997 Phys. Rev. Lett. 791217 ibid 1998 Phys. Rev. D57 2957

[5] Li C S, Oakes R J and Yang J M 1994 Phys. Rev. D49 293 Atwood D, Reina L and Soni A 1995 Phys. Rev. Lett. 753800 Bar-Shalom S, Atwood D, Eilam G, Mendel R R and Soni A 1996 Phys. Rev. D53 1162 Hou W S and Lin G L 1996 Phys. Lett. B379 261 Mahanta U and Ghosal A 1998 Phys. Rev. D57 1735 Bar-Shalom S, Eilam G and Soni A 1999 Phys. Rev. D59 055012 Yu Z H et al 2000 Eur. Phys. J. C16 541 Chemtob M and Moreau G 1999 Phys. Rev. D59 116012

[6] Han T and Hewett J L 19999 Phys. Rev. D60 074015

[7] Peccei R D and Zhang X 1990 Nucl. Phys. B337 269 Han T, Peccei R D and Zhang X 1995 Nucl. Phys. B434 527 Han T, Whisnant K, Young B L and Zhang X 1997 Phys. Rev D55 7241 Bar-Shalom S and Wudka J 1999 Phys. Rev. D60 094016 Tait T and Yuan C P 2001 Phys. Rev. D63 014018 Alan A T and Senol A 2002 Europhys.Lett. 59 669-673

[8] Assmann R W et al 2000 The CLIC Study Team CERN 2000-008

[9] TESLA Technical Design Report (2001) DESY 2001-011

[10] Groom D E et al 2000 Eur. Phys. J. C15 1 


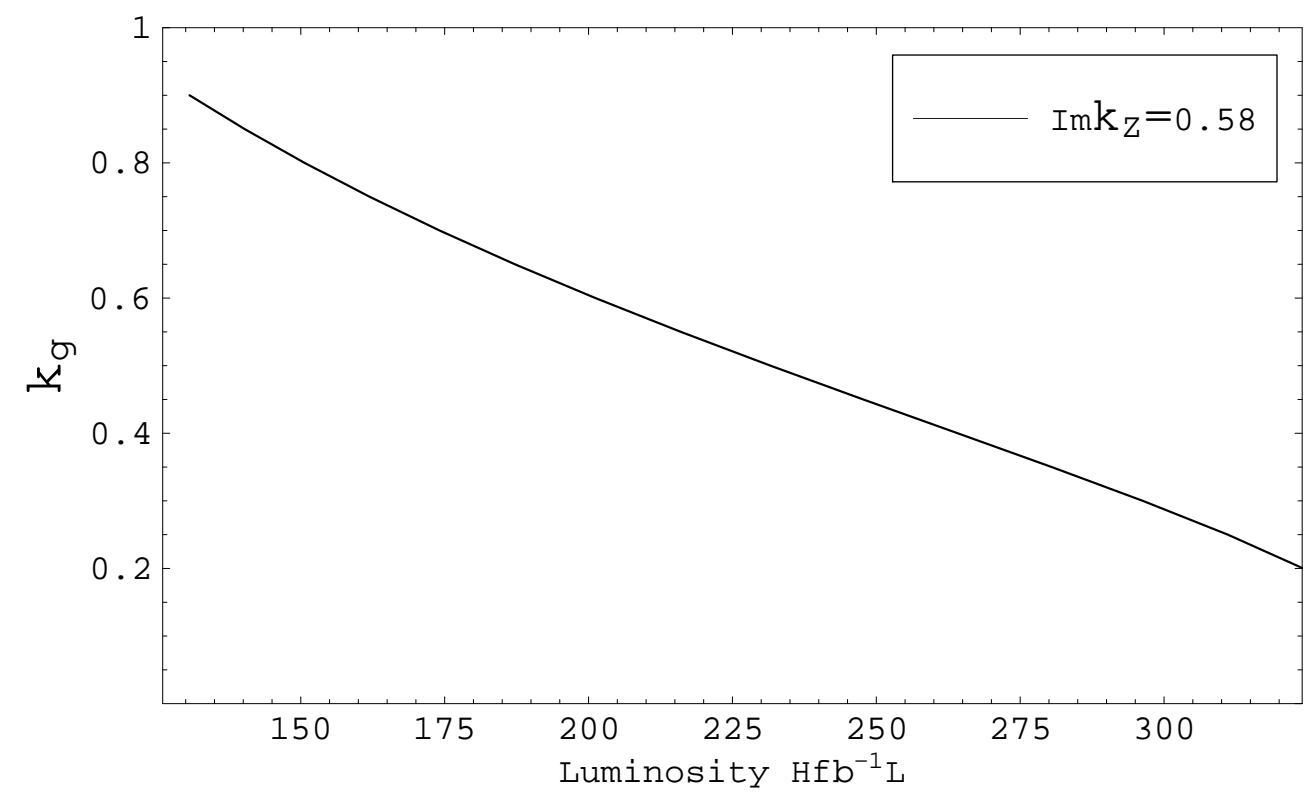

(a)

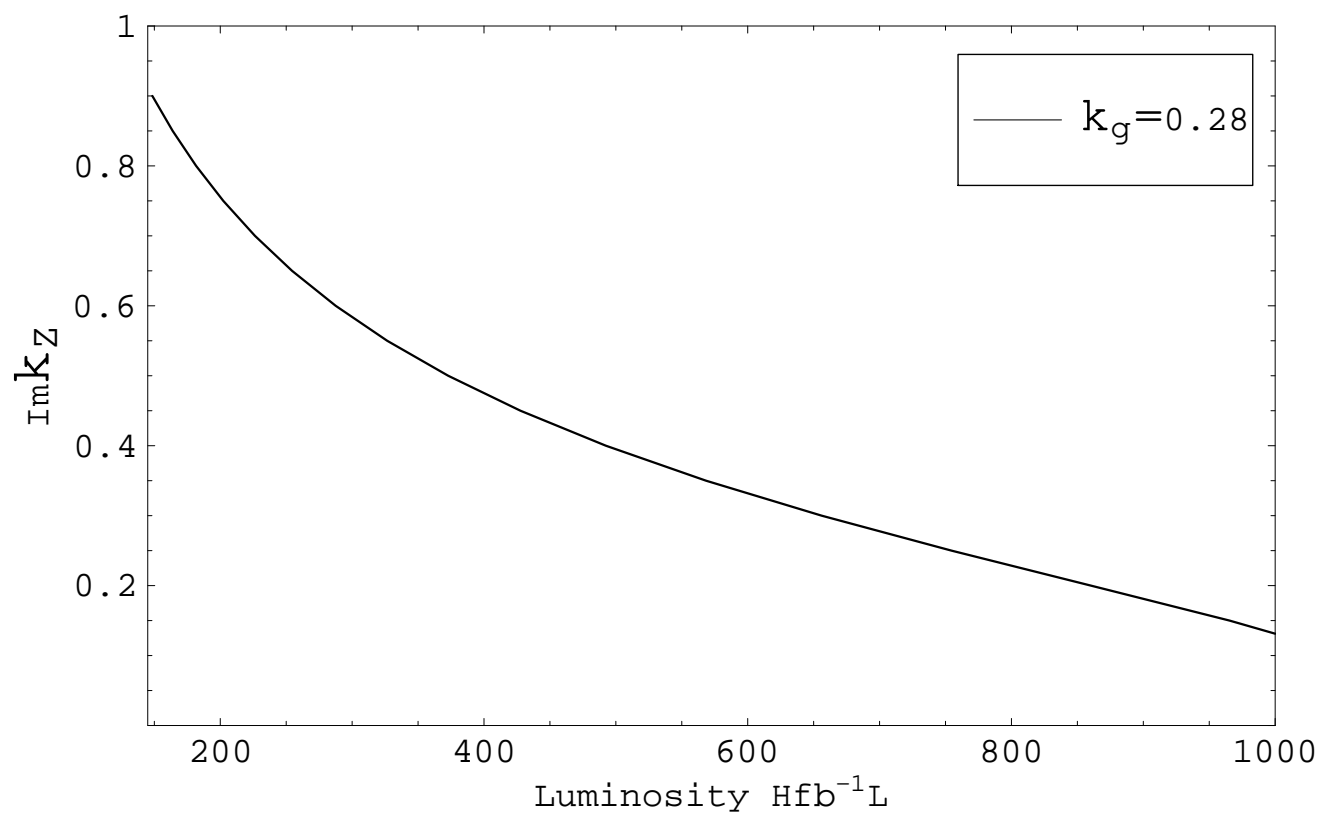

(b)

Figure 1. 95\% C.L. sensitivity to the FCNC couplings as a function of the integrated luminosity for $\sqrt{s}=500 \mathrm{GeV}$. 
CP violating asymmetries in the flavour changing single top quark production

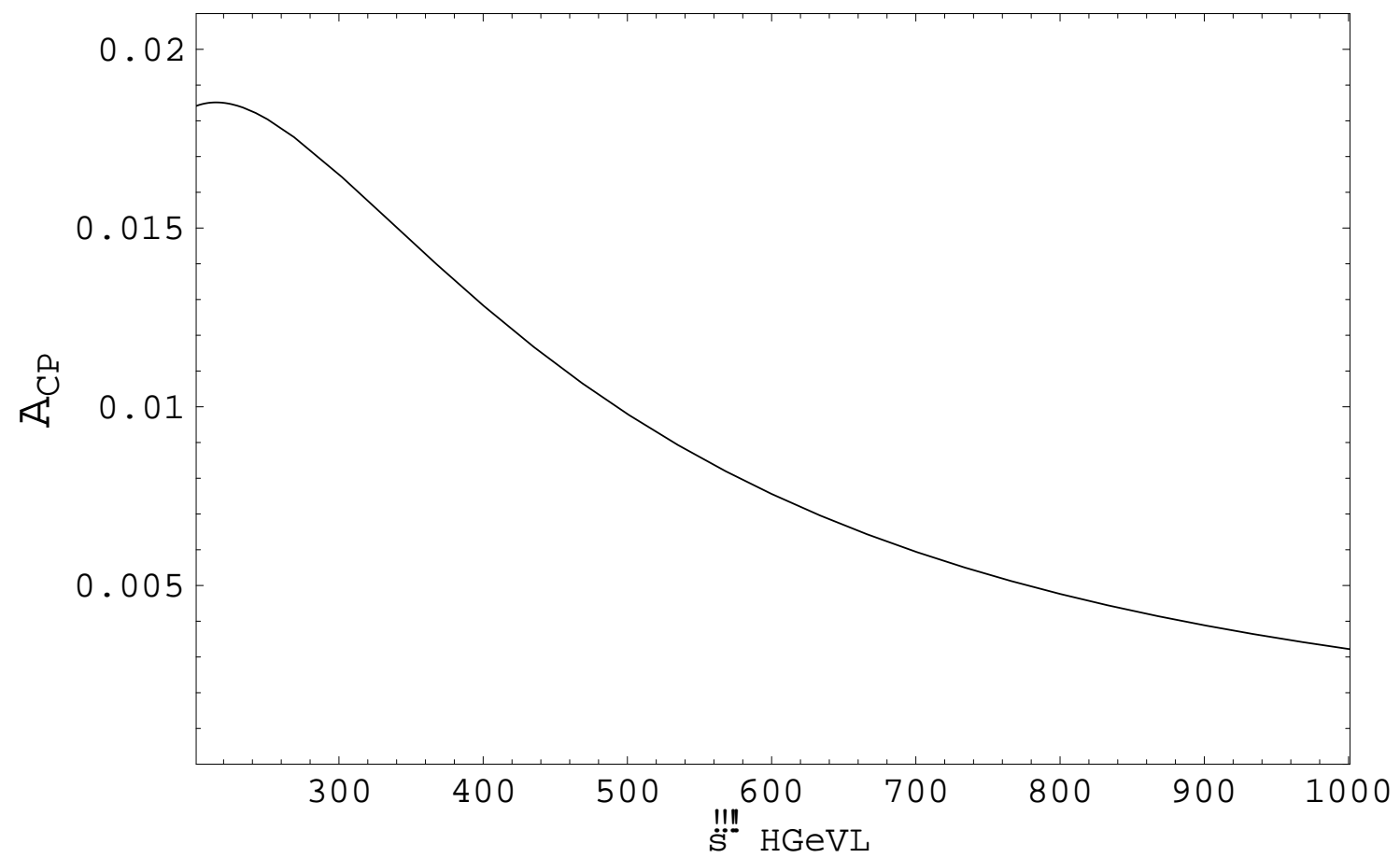

Figure 2. CP asymmetry as a function of the CM energy with $\tilde{\kappa}_{\gamma}=0.28$ and $\operatorname{Im} \tilde{\kappa}_{Z}=0.58$.

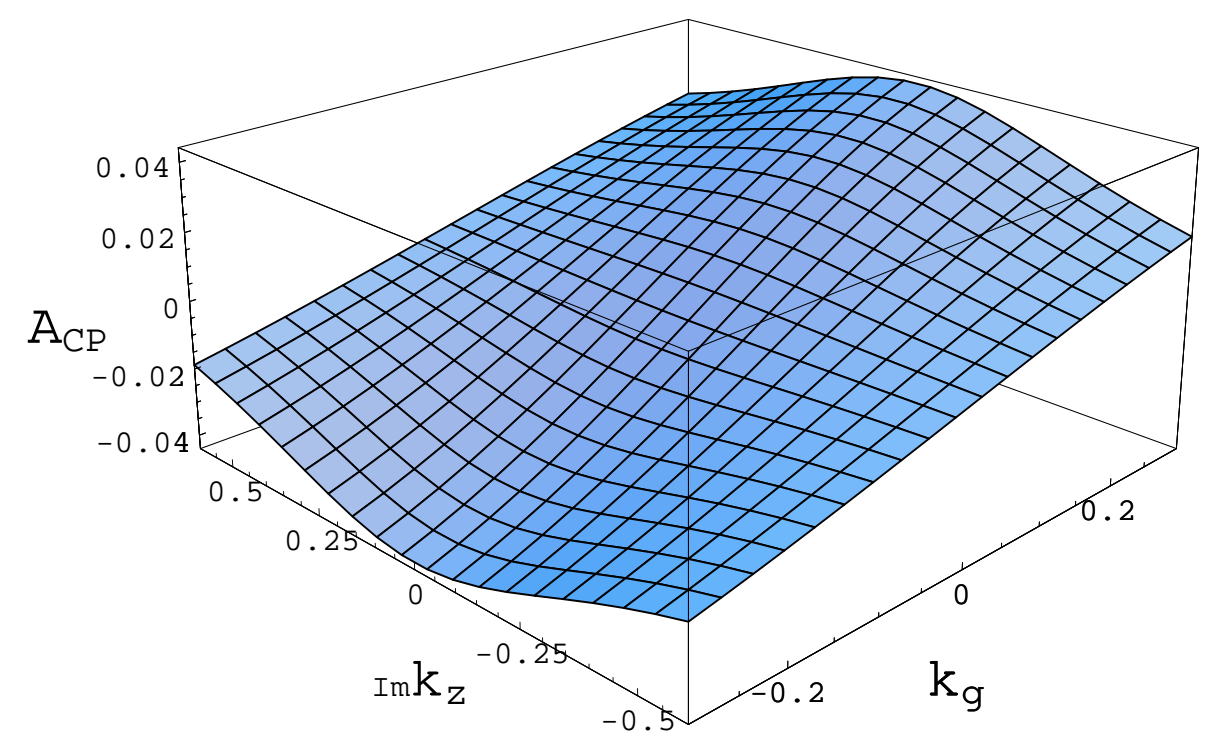

Figure 3. CP asymmetry as a function of $\tilde{\kappa}_{\gamma}$ and $\operatorname{Im} \tilde{\kappa}_{Z}$ at $\sqrt{s}=200 \mathrm{GeV}$. 


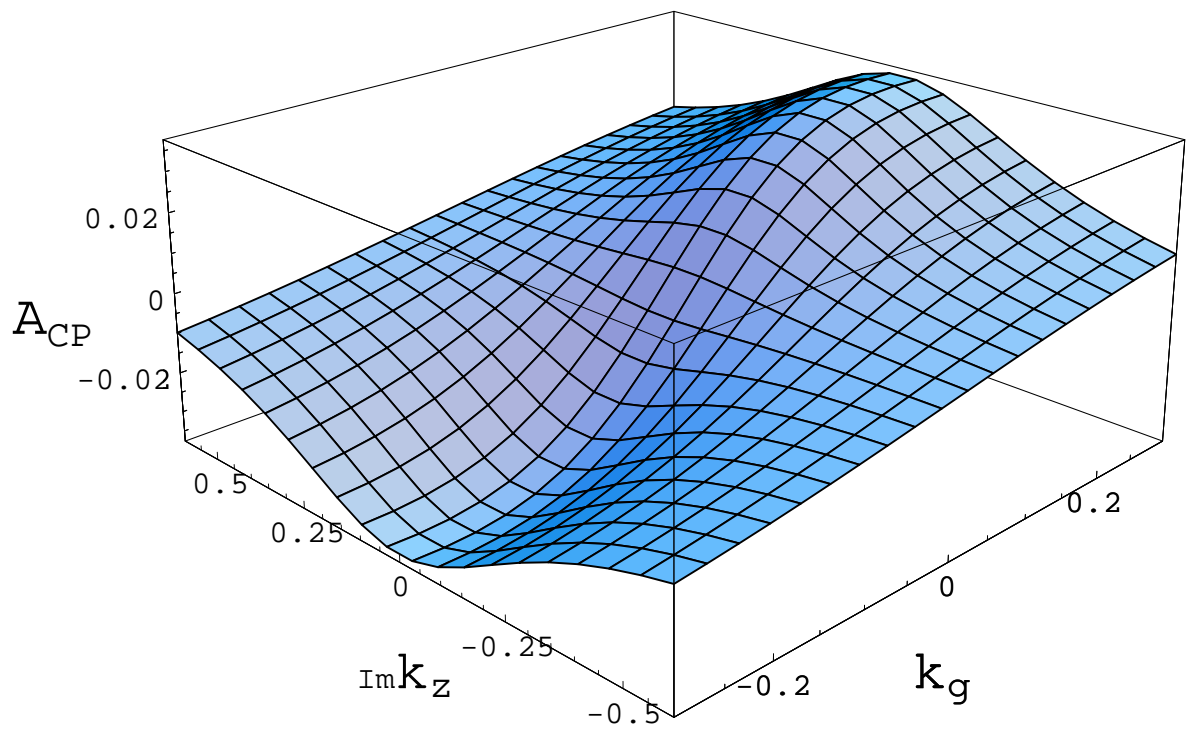

Figure 4. CP asymmetry as a function of $\tilde{\kappa}_{\gamma}$ and $\operatorname{Im} \tilde{\kappa}_{Z}$ at $\sqrt{s}=500 \mathrm{GeV}$.

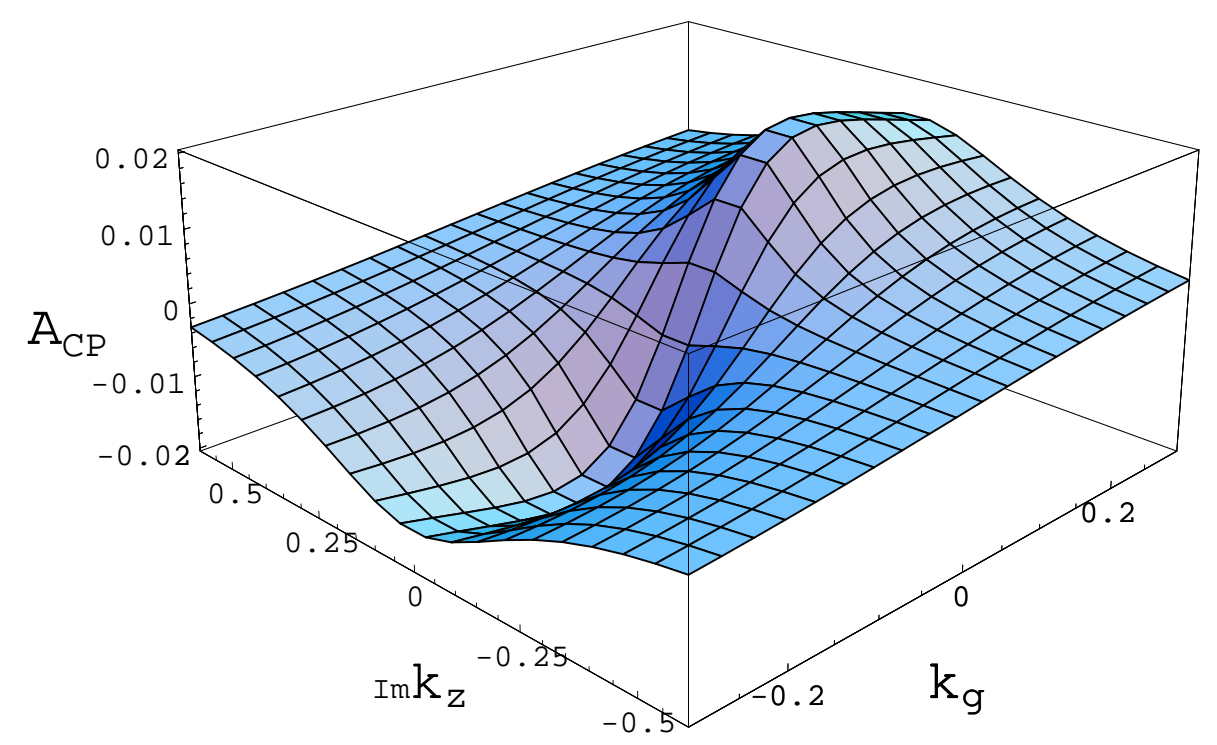

Figure 5. CP asymmetry as a function of $\tilde{\kappa}_{\gamma}$ and $\operatorname{Im} \tilde{\kappa}_{Z}$ at $\sqrt{s}=1000 \mathrm{GeV}$. 


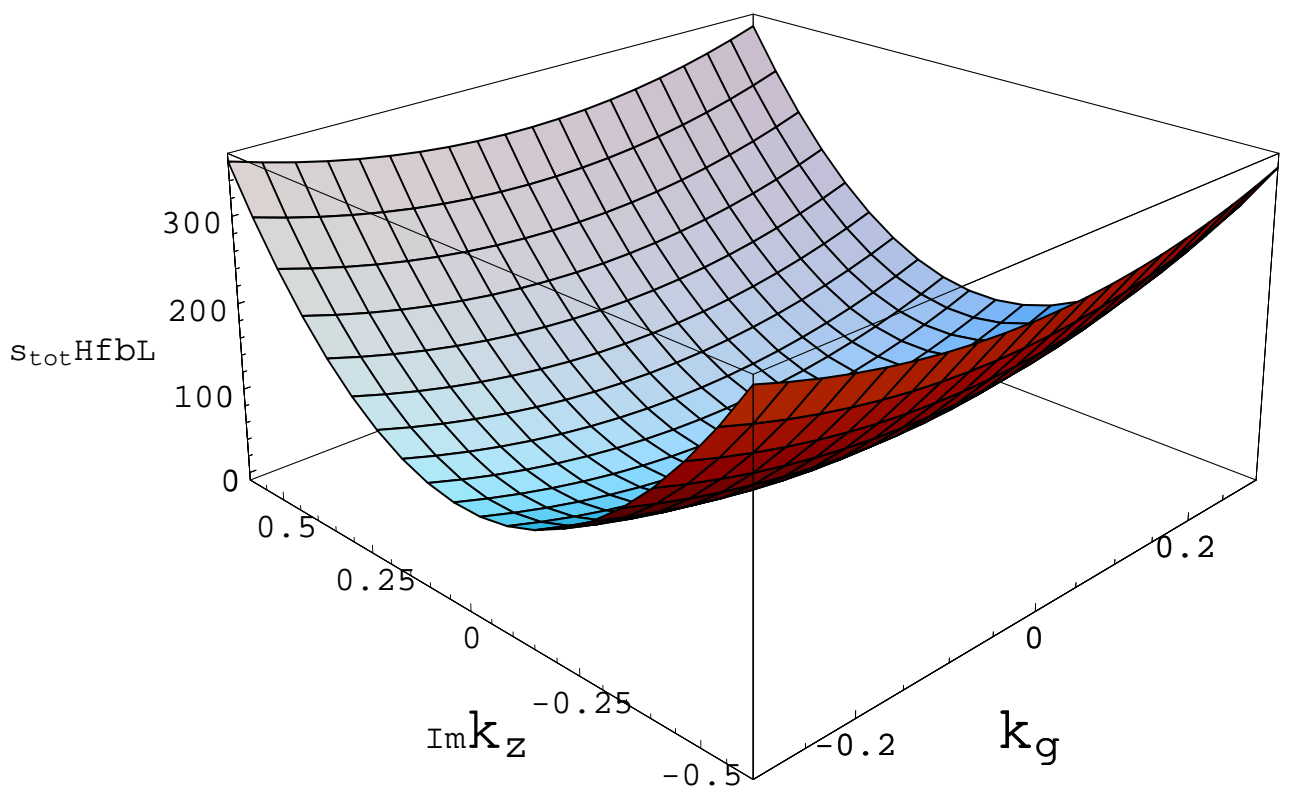

Figure 6. The total cross-section for $e^{+} e^{-} \rightarrow t \bar{q}$, with $\sqrt{s}=500 \mathrm{GeV}$, as a function $\tilde{\kappa}_{\gamma}$ and $\operatorname{Im} \tilde{\kappa}_{Z}$. 